American Journal of Biochemistry and Biotechnology 4 (2): 105-113, 2008

ISSN 1553-3468

(C) 2008 Science Publications

\title{
Altered Sulfur Amino Acid Metabolism In Immune Cells of Children Diagnosed With Autism
}

\author{
${ }^{1,3}$ Jung H. Suh, ${ }^{2}$ William J. Walsh, ${ }^{2}$ Woody R. McGinnis, ${ }^{2}$ Allen Lewis, and ${ }^{1,3}$ Bruce N. Ames \\ ${ }^{1}$ Children's Hospital Oakland Research Institute, Oakland, CA \\ ${ }^{2}$ Pfeiffer Treatment Center, Warrenville, Il \\ ${ }^{3}$ Children's Hospital Oakland Research Institute, 5700 Martin Luther King Jr. Way \\ Oakland, CA 94609, USA
}

\begin{abstract}
Autism Spectrum Disorder (ASD) is a behaviorally defined neurodevelopmental disorder whose etiology is poorly understood. Recent studies have shown that autistic children may be experiencing increased inflammation and oxidative stress. Altered immune regulation may be one contributing factor to inflammation and oxidative stress in autistic children. Sulfur amino acid (SAA) metabolism plays a critical role in regulating blood leukocyte functions and oxidative stress. However, it is not known whether autism impacts SAA metabolism in peripheral immune cells. To address this question, a novel liquid chromatography linked tandem mass spectrometric (LC/MS/MS) method was used to determine the levels of SAA metabolites in peripheral blood mononuclear cells obtained from 11 healthy controls and 31 autistic children. Improved detection sensitivity and selectivity of the LC/MS/MS method allowed accurate quantification using small samples. Results show that leukocytes from autistic children contained significantly lower concentrations of S-adenosylmethionine $(-35 \% ; \mathrm{p}$ $=0.01)$, and elevated levels of intracellular homocysteine content $(+80 \% ; \mathrm{p}=0.003)$. Additionally, the levels of intracellular total cysteine and glutathione $(\mathrm{GSH})$ were reduced by $39 \%(\mathrm{p}=0.004)$ and $25 \%$ $(\mathrm{p}=0.01)$, respectively. These autism-associated changes were leukocyte specific in that no significant alterations in SAA metabolite concentrations were detected in the plasma samples. Our results provide novel evidence for altered metabolism in immune cells; furthermore, this data suggest the involvement of inflammation in autism. Dietary differences between controls and patients, however, remain a potential confounder.
\end{abstract}

Key words: Autism, Sulfur Amino Acids, Metabolism, Oxidative Stress, Inflammation, and Leukocytes

\section{INTRODUCTION}

Autism spectrum disorder (ASD) is a neurodevelopmental disorder with no established genetic or biochemical biomarkers. ASD is diagnosed by language impairment, impaired social interactions, and a tendency to exhibit repetitive behavior ${ }^{[1]}$. It is unclear as to whether the heterogeneity of these clinical symptoms is reflective of different manifestations of a single disease or whether these observations indicate many discrete diseases with distinct etiologies. A recent Center for Disease Control (CDC) report estimates that 1 in 150 children are afflicted by this disorder, making autism one of the most prevalent childhood neurodevelopmental disorders ${ }^{[2]}$. However, it is also not clear whether the dramatic rise in the incidence of autism within the last decade is due to the broadening of the definition of autism, increased surveillance and awareness, ${ }^{[3]}$ or to an actual increase in the incidence of the disorder ${ }^{[4]}$.

Emerging data now suggest that altered immune regulation may be a component of autism phenotype. Evidence of systemic and localized central nervous system (CNS) inflammation has been found in children with ASD. Enhanced microglial and astroglial activation and elevated levels of proinflammatory cytokines were detected in the brain and in the cerebrospinal fluids of children with ASD ${ }^{[5]}$.

Additionally, decreased mitogenic responses of circulating $\mathrm{T}$ cells ${ }^{[6]}$ and excessive innate immune cell activation ${ }^{[7-9]}$ have been detected in ASD patients. Cytokine profiles in peripheral blood also show

Corresponding Author: $\quad$ Bruce N. Ames and Jung H. Suh, Children's Hospital Oakland Research Institute 5700 Martin Luther King Jr. Way, Oakland, CA 94609, USA 
evidence of elevated levels of the pro-inflammatory cytokines, IL-4, and IL-6 ${ }^{[10] . ~ M o r e o v e r, ~ i t ~ h a s ~ b e e n ~}$ shown that autism may be associated with a decline in cell-mediated adaptive immunity, as indicated by reports of decreases in Th1-related cytokines such as IL-2 and IFN- $\gamma^{[11]}$. The underlying mechanism(s) for autism-associated immune dysfunction has yet to be identified.

Heightened oxidative stress is a component of many chronic inflammatory diseases. Inflammation is associated in enhanced cellular oxidant production ${ }^{[12,13]}$ which in turn, can cause oxidative damage to lipids, proteins and DNA. Studies have found that urinary and plasma levels of lipid oxidation markers in autistic children were significantly elevated in comparison to healthy controls ${ }^{[14,15]}$. In addition, children with autism were found to have lower plasma glutathione concentrations than their age- and gender-matched healthy controls ${ }^{[16,17]}$. The reported autism-related increase in lipid oxidation and loss of low molecular weight antioxidants suggest that autism is associated with increased oxidative stress ${ }^{[14,18,19] \text {. }}$

Sulfur amino acids (SAA) are essential for maintaining optimal immunity and systemic redox homeostasis. SAA metabolism via the transmethylation, transsulfuration and GSH synthesis pathways produces key metabolites required to support immune cell function and intracellular antioxidant defense ${ }^{[20]}$. Interfering with any one of these pathways can disrupt lymphocyte proliferation ${ }^{[21]}$, cytokine synthesis ${ }^{[22]}$ and impair intracellular redox homeostasis ${ }^{[23]}$.

Three studies report altered SAA metabolism in autistic children. In a study involving 12 autistic and 9 healthy control children, Pasça et al. found significant elevation in plasma homocysteine levels ${ }^{[24]}$. A more comprehensive analysis of SAA metabolism was performed in studies published by James et al ${ }^{[16,17]}$. The results from these studies show that in comparison to healthy controls, key metabolites derived from the transmethylation, transsulfuration, and glutathione synthesis pathways were decreased in autistic children ${ }^{[16,17]}$. However, unlike the study by Pasça et al. ${ }^{[24]}$, James et al. do not report any autism-related increases in plasma homocysteine ${ }^{[16,17]}$. Despite this discrepancy regarding one metabolite, data from these studies suggest that altered SAA metabolism may be a common autism phenotype. However, the underlying mechanism(s) contributing to these changes in SAA metabolism in autistic children remains unclear.

In a recent study, Yao and co-workers ${ }^{[25]}$ measured urinary concentrations of isoprostane $\mathrm{F}_{2 \alpha}$-VI and 2,3dinor-thromboxane $\mathrm{B}_{2}{ }^{[25]}$. These are sensitive markers of lipid peroxidation and platelet activation, respectively ${ }^{[25]}$. This study was unique in that none of the control and ASD children were supplemented with any nutritional supplements or prescription medications for at least six months prior to the collection of the sample data. Their results showed that relative to healthy controls, autistic children had significantly elevated concentrations of urinary isoprostane $\mathrm{F}_{2 \alpha}$-VI and 2,3-dinor-thromboxane $B_{2}$, suggesting that the presence of heightened inflammation and oxidative stress in this group of autistic children ${ }^{[25]}$.

Using the plasma and peripheral blood leukocytes obtained from this cohort, we sought to examine whether SAA metabolism in the leukocytes was affected in autistic children. To this end, we used a sensitive high throughput liquid chromatography-linked tandem mass spectrometric (LC/MS/MS) technique that measures the small molecule metabolites involved in the transmethylation, transsulfuration, and GSH synthesis pathways. Plasma metabolite content was also measured to determine if the differences in immune cell SAA metabolites were due to changes in substrate bioavailability.

Prior to this study, SAA metabolism in peripheral leukocytes in autistic children had not been examined. A finding of altered SAA metabolism in peripheral mononuclear cells in this population would be further evidence of a possible role of inflammation and oxidative stress in the etiology of autism; furthermore, such findings could suggest new areas of investigation for therapeutic interventions.

\section{MATERIALS AND METHODS}

Study Subjects: Subject recruitment and blood sample collection from 11 healthy controls and 31 children diagnosed with ASD were performed at the Pfeiffer treatment center as part of a pilot study. Sample collection included urine samples collected by the study done by Yao et al ${ }^{[25]}$. The diagnosis of ASD was made using criteria provided by the Diagnostic and Statistical Manual of Mental Disorders, Fourth Edition (DSM IV) and by the Autism Diagnostic Interview - Revised (ADI-R).

Children with ASD were excluded from the study if they: 1) Had any nutritional or antioxidant supplementation in the period 6 mo prior to the beginning of the study, 2) Had any pre-existing chronic illness (pulmonary, vascular or other mental diseases) 3) Had acute sickness within 2 weeks prior to sample collection, and 4) were receiving psychiatric, antiinflammatory or chelation therapy.

Control subjects were included if they: 1) Had no family history of autism, 2) Were not receiving any 
medications or therapy, 3) Had no history of mental disorder, and 4) Had no nutritional or antioxidant supplementation. The institutional review board of the Pfeiffer Treatment Center approved the study and the informed consents were obtained from the participant families.

Materials: Methionine (Met), methionine sulfoxide (MetSO), S-adenosylmethionine (SAM), Sadenosylhomocysteine (SAH), homocysteine (Hcy), homocystine (Hcy2), cystathionine (Cysth), cysteine (Cys), cystine (Cys2), glutathione (GSH), glutathionedisulfide (GSSG), cysteinyl-glycine (cysgly), cysteinylglycine disulfide (Cysgly2), glycine (Gly), glutamine (Gln), and glutamate (Glu) of highest analytical grade were purchased from Sigma (St. Louis, MO). Homoglutathione was purchased from BACHEM. Stable isotopes, methionine-methyl- $\mathrm{D}_{3}$, Homocystine $\left(3,3,3^{\prime}, 3^{\prime}, 4,4,4^{\prime}, 4^{\prime}-\mathrm{D}_{8}\right)$, Cystine $\left(3,3,3^{\prime} 3^{\prime}-\mathrm{D}_{4}\right)$, and Glycine $\left({ }^{13} \mathrm{C}_{2},{ }^{15} \mathrm{~N}\right)$ were purchased from Cambridge Isotopes (Andover, MA). S-Adenosylmethionine (methyl-D $_{3}$ ) was obtained from CDN Isotopes (Quebec, Canada). All HPLC solvents were of the analytical grade available from Fisher scientific. Materials used for derivatization and HPLC reverse phase columns were part of the EZ-FAAST kit available from Phenomenex (Torrance, CA).

Blood collection: Blood (7 $\mathrm{ml})$ was collected by venipuncture to the antecubital vein with 23-gauge needle into $\mathrm{K}_{2}$ EDTA tubes. These samples were centrifuged at $800 \times \mathrm{g}$ for $10 \mathrm{~min}$. Subsequently, plasma and buffy coat samples were collected and stored at $-80 \mathrm{C}$.

Plasma sample preparation: For the analysis of total sulfur amino acids, $150 \mu \mathrm{l}$ of plasma obtained were incubated with $150 \mu \mathrm{l}$ of $50 \mathrm{mM}$ dithiothreitol (DTT) containing $1 \mathrm{mM}$ potassium oxalate at room temperature for $10 \mathrm{~min}$ to completely reduce plasma mixed disulfides. DTT treated plasma samples $(150 \mu \mathrm{l})$ were then mixed with equal volume of PCA solution, vortexed and centrifuged at $13,000 \mathrm{rpm}$ for $5 \mathrm{~min}$. The acid soluble supernatants were used for quantifying total plasma SAA metabolites. To reduce the potential variability due to ex-vivo oxidation of sulfhydrylcontaining metabolites, all plasma samples were reduced with dithiothreitol prior to analysis.

Peripheral blood mononuclear cells (PBMC): Contaminating erythrocytes in buffy coat samples were removed by hypotonic lysis. Briefly, $0.2 \mathrm{ml}$ of buffy coat samples were mixed with $5 \mathrm{ml}$ of $0.15 \mathrm{M}$ ammonium chloride solution containing $10 \mathrm{mM}$ potassium bicarbonate, and $0.1 \mathrm{mM}$ EDTA and incubated for $5 \mathrm{~min}$ at room temperature. Samples were then centrifuged at $2000 \times \mathrm{g}$ for $10 \mathrm{~min}$ to pellet intact cells. The resultant PBMC cell pellet was washed twice with $10 \mathrm{mM}$ potassium buffered saline. The washed PBMC cells were resuspended in $5 \%$ perchloric acid containing $1 \mathrm{mM}$ DTPA and centrifuged at 13,000 rpm for $5 \mathrm{~min}$. The acid-soluble supernatants were collected for LC/MS/MS analysis. The remaining protein pellets were dissolved and used for protein quantification using the Bradford assay.

High Pressure Liquid Chromatography linked tandem mass spectrometry (LC/MS/MS): SAA metabolites (see supplementary table 1) were detected using LC/MS/MS following derivatization with isopropylchloroformate based on methods previously published by Husek et al., [26] with the following modifications. The $200 \mu \mathrm{l}$ acid-soluble supernatants collected above were mixed with internal standards (see supplementary table 1., $10 \mu \mathrm{M}$ final conc.). Subsequently, solid phase extractions with a strong cation exchange resin (Phenomenex) were performed. SAA metabolites were eluted by treating with a buffer $(200 \mu \mathrm{l})$ consisting of $0.1 \mathrm{~N} \mathrm{NaOH}, 40 \% \mathrm{~N}$-propanol, and $10 \%$ pyridine. The eluted samples were mixed with $50 \mu \mathrm{l}$ of derivatizing solution consisting of isooctane, chloroform, and isopropylchloroformate (75:40:10) and allowed to react for $2 \mathrm{~min}$. The derivatized products were subsequently extracted with $100 \mu$ l of isooctane and dried under a constant stream of nitrogen. These samples were reconstituted with $100 \mu \mathrm{l}$ of mobile phase consisting of methanol and water (80:20, v/v).

Chromatographic separation of SAA metabolites was performed on C18 reversed-phase column $(250 \times 20$ $\mathrm{mm}, 4 \mu \mathrm{m}$ ) from Phenomenex (Torrance, Ca). The chromatographic system was Shimadzu LC-10AV separation module. The separation was performed under isocratic condition at a flow rate of $0.2 \mathrm{ml} / \mathrm{min}$.

Electrospray tandem mass spectrometric analysis was performed on Quattro Micro mass spectrometer from Micromass and analyzed using Mass Lynx software (3.1 version). Analytes were detected using the multiple reaction monitoring (MRM) scanning mode. Capillary voltage was set to $3 \mathrm{kV}$, source temperature to $150{ }^{\circ} \mathrm{C}$ and nebulizer gas temperature to $400{ }^{\circ} \mathrm{C}$. The cone and evaporation gas flows were set at 100 and $800 \mathrm{~L} / \mathrm{hr}$, respectively. The specific cone voltage and collision energy used for each metabolites are provided in the table 1, which also lists all of the internal standards used for quantification. 
Statistical analysis: Differences in the means for individual metabolites between control and ASD afflicted children were examined using the student's $t$ test. Analysis was performed using SPSS 14.0 for Windows (SPSS In., Chicago, IL).

\section{RESULTS AND DISCUSSION}

General subject profile: A total of 11 healthy and 31 autistic children were recruited into the study. The mean age of the children in the control group was $6.9 \pm$ $1.6 \mathrm{yrs}$, whereas the mean age in autistic group was 4.2 \pm 1.3 (Table 2). The male to female ratios were similar in the two groups. Questionnaires were sent to the parents of the children afflicted with ASD, and 28 responses were received. Two of the responses were incomplete regarding problems with pica. Based on the responses, the median age of diagnosis was at $21 \pm 6.5$ mo of age. As reported by the parents, 21 out of the 28 children suffered from varying degrees of GI symptoms, which ranged from loose stools to frequent diarrhea. Parents indicated that 10 out of the 26 children had problems with pica. Approximately $40 \%$ of the ASD children displayed a delay in motor development and $64 \%$ displayed delayed speech development. Approximately $67 \%$ of ASD-afflicted children displayed tendencies to avoid eating vegetables, while $53 \%$ tended to avoid fruits. However, since questionnaires were not sent to the control families, the prevalence of the above listed problems in the controls is unknown.

Transmethylation pathway metabolites in leukocytes: The transmethylation pathway begins with the metabolic conversion of the essential amino acid methionine (Met) to S-adenosylmethionine (SAM). Utilization of SAM for methyl transfer reactions leads to the formation of S-adenosylhomocysteine (SAH), which is subsequently hydrolyzed to form homocysteine (Hcy). The homocysteine formed can be either re-methylated to form Met or enter into the transsulfuration pathway to form cystathionine.

Transmethylation metabolites in PBMC obtained from ASD-afflicted children displayed significant differences compared to the controls. Specifically, the mean PBMC SAM levels were 36\% ( $\mathrm{p}=0.03)$ lower in the ASD group $(0.028 \pm 0.02 \mathrm{nmol} / \mathrm{mg}$ protein $)$ compared to controls $(0.044 \pm 0.02 \mathrm{nmol} / \mathrm{mg}$ protein; Figure 1B). This resulted in a significant $50 \%$ decrease in the $\mathrm{SAM} / \mathrm{SAH}$ ratio in PBMC from ASD children versus healthy control children, indicating a loss in cellular methylation capacity (Figure 1C). Associated with this loss, the total PBMC homocysteine content was markedly elevated in the ASD children (0.028 \pm $0.025 \mathrm{nmol} / \mathrm{mg}$ protein) relative to controls $(0.01 \pm$ $0.004 \mathrm{nmol} / \mathrm{mg}$ protein; Figure $1 \mathrm{E})$. The above results are likely to have a major negative impact on lymphocyte proliferation and oxidative stress. The rate of SAM synthesis by methionine adenosyltransferase (MAT, EC 2.5.1.6) is markedly elevated in rapidly proliferating lymphocytes to meet the enhanced demand for methylation ${ }^{[27,28]}$ and for polyamine synthesis ${ }^{[29] .}$ It is possible that low levels of SAM may be a reflection of the decreased lymphocyte mitogenic response detected in some autistic children ${ }^{[6]}$. Accumulation of intracellular Hcy may directly contribute to heightened oxidative stress in lymphocytes. Hcy is highly oxidizing and exists predominantly as protein-mixed disulfides, both inside and outside cells. In vitro, Hcy directly inhibits intracellular metallothioneine (MT), by preferentially forming mixed disulfides with MT ${ }^{[30]}$. Exogenous addition of Hcy $(0.3$ to $3 \mathrm{mM})$ in vitro has also been shown to increase the rate of oxidant generation $^{[31,32]}$.

Transsulfuration and GSH synthesis pathway metabolites: Hcy can supply cysteine required for GSH synthesis via the transsulfuration pathway. The metabolic fate of Hcy is regulated, in part, by the availability of SAM. Elevated intracellular SAM acts as an allosteric inhibitor of methylenetetrahydrofolate reductase (MTHFR, EC 1.5.1.20) and enhances the activity of cysthathionine $\beta$ synthase. The observed loss in SAM (Figure 1B.) may lead to decreased formation of cystathionine and limit its conversion to cysteine ${ }^{[23]}$. We observed that the total intracellular cysteine (cysteine +2 cystine) level was significantly ( $\mathrm{p}=$ $0.004)$ lower in children afflicted with ASD (0.16 \pm $0.11 \mathrm{nmol} / \mathrm{mg}$ protein) relative to the controls $(0.27 \pm$ 0.12 ; Figure $2 \mathrm{~A})$. Concomitant with a decrease in cysteine, GSH concentration $(1.03 \pm 0.13 \mathrm{nmol} / \mathrm{mg}$ protein) was lower in ASD children than when compared to controls $(1.63 \pm 0.21 \mathrm{nmol} / \mathrm{mg}$ protein; $\mathrm{p}=0.02$; Figure 2B). While differences were not significant, total GSH (GSH+2 GSSG) levels in autistic children trended lower than the controls (Figure 2D). Similar patterns were observed with GSH redox state (Figure 2E). No alterations in Cysgly levels were detected between the ASD and control groups (Figure $2 \mathrm{~F})$.

A decline in leukocyte GSH concentrations is likely to compromise immune functions in a number of 
different ways. As the principal redox buffer, intracellular GSH level is a critical determinant of lymphocyte proliferation ${ }^{[21]}$. In vivo depletion of GSH is associated with a reduced number of activated $\mathrm{T}$ cells and decreases the rate of IL-2 synthesis ${ }^{[22]}$. Loss of GSH may also promote lymphocyte apoptosis ${ }^{[22]}$ and contribute to the observed imbalance between Th-1/Th2 responses reported in autism patients ${ }^{[6,8,33,34]}$.

GSH decrease in autistic children may arise from either enhanced rate of its utilization, export and/or decreases in the cellular synthesis rate. Significant decreases in intracellular Cys needed for GSH synthesis suggest that the rate of Cys import might not be adequate to maintain normal GSH levels. The increased oxidative stress observed in the leukocytes of autistic children might increase the demand for GSH, though this remains to be shown.
Plasma SAA substrate and metabolite levels: In order to determine whether the observed differences in PBMC SAA metabolite concentrations was due to lack of substrate availability, the corresponding plasma samples were analyzed. Plasma Cysgly in the ASD children $(17.5 \pm 6.8 \mu \mathrm{M})$ were significantly higher in comparison to controls $(10.5 \pm 4.1 \mu \mathrm{M})$. This suggests an increase in either hepatic or serum activity of $\gamma$ Glutamyltranspeptidase (GGT, EC 2.3.2.2) in children diagnosed with autism (Table 3).

The elevated plasma Cysgly content in ASD children is evidence of heightened inflammatory stress in autism. Cysgly is formed by enzymatic hydrolysis of GSH mediated by GGT, which becomes elevated during inflammation [35,36]. A rise in GGT enhances the rate by which GSH is recycled; thus, the elevation of GGT may be a compensatory response to the rise in systemic oxidative stress.

Table 1: Mass Spectrometry Parameters

\begin{tabular}{|c|c|c|c|c|c|c|}
\hline No & Compound & {$[\mathrm{MH}]^{+}$} & Transition Pairs & Cone (V) & Collision $(\mathrm{eV})$ & Internal STI \\
\hline 1 & Methionine sulfoxide & 294.3 & $294.3 \rightarrow 234.3$ & 33 & 10 & 2 \\
\hline 2 & Methionine sulfoxide (methyl- $\mathrm{D}_{3}$ ) & 297.3 & $297.3 \rightarrow 237.5$ & 33 & 10 & IS \\
\hline 3 & S-Adenosylmethionine & 699.3 & $699.3 \rightarrow 230$ & 35 & 18 & 4 \\
\hline 4 & S-Adenosylmethionine (methyl- $\mathrm{D}_{3}$ ) & 702.3 & $702.3 \rightarrow 230$ & 35 & 18 & IS \\
\hline 5 & Cysteinylglycine & 393.5 & $393.5 \rightarrow 248.5$ & 30 & 12 & 13 \\
\hline 6 & Cysteinylglycine disulfide & 611.5 & $611.5 \rightarrow 363.5$ & 30 & 20 & 13 \\
\hline 7 & Methionine & 278.0 & $278 \rightarrow 190$ & 20 & 15 & 8 \\
\hline 8 & Methionine (methyl- $\mathrm{D}_{3}$ ) & 281.0 & $281 \rightarrow 193$ & 20 & 15 & IS \\
\hline 9 & S-adenosylhomocysteine & 611.5 & $611.5 \rightarrow 363.5$ & 30 & 20 & 4 \\
\hline 10 & Cystathionine & 479.2 & $479.2 \rightarrow 230.5$ & 35 & 16 & 13 \\
\hline 11 & Cysteine & 336.5 & $336.5 \rightarrow 190.3$ & 30 & 12 & 13 \\
\hline 12 & Cystine & 497.5 & $497.5 \rightarrow 248.5$ & 37 & 17 & 13 \\
\hline 13 & Cystine $\left(3,3,3^{\prime} 3^{\prime}-\mathrm{D}_{4}\right)$ & 501.5 & $501.5 \rightarrow 250.5$ & 37 & 17 & IS \\
\hline 14 & Glutathione & 564.5 & $564.5 \rightarrow 162.2$ & 30 & 20 & 19 \\
\hline 15 & Glutathione disulfide & 953.5 & $953.5 \rightarrow 836.5$ & 58 & 20 & 19 \\
\hline 16 & Homocysteine & 350.0 & $350 \rightarrow 204$ & 30 & 13 & 18 \\
\hline 17 & Homocystine & 525 & $525 \rightarrow 262$ & 30 & 12 & 18 \\
\hline 18 & Homocystine $\left(3,3,3^{\prime}, 3^{\prime}, 4,4,4^{\prime}, 4^{\prime}-\mathrm{D}_{8}\right)$ & 533.5 & $533.5 \rightarrow 266.5$ & 35 & 20 & IS \\
\hline 19 & Homoglutathione & 578.5 & $578.5 \rightarrow 162.2$ & 30 & 20 & 3 \\
\hline
\end{tabular}

a IS marks the internal standards used for quantification. The number denotes the specific IS compound number used for a given compound

Table 2: Study Subjects

\begin{tabular}{llll} 
& Control & ASD & $\mathrm{p}$ \\
\hline No. of subjects & 11 & 31 & $<.05$ \\
Age & $6.9 \pm 1.6^{\mathrm{a}}$ & $4.17 \pm 1.3^{\mathrm{a}}$ & $\mathrm{ns}$ \\
Gender (M/F) & $9 / 2$ & $26 / 4$ & \\
Age at diagnosis (mo) & & $21 \pm 6.5$ & $70.0 \%(21 / 28)^{\mathrm{b}}$ \\
GI problems & & $38.5 \%(10 / 26)^{\mathrm{b}}$ & \\
Pica & & $40.7 \%(11 / 27)^{\mathrm{b}}$ & \\
Delayed Motor Development & & $64.3 \%(18 / 28)^{\mathrm{b}}$ & \\
Delayed Speech & & & \\
Development & & & \\
\hline
\end{tabular}

${ }^{\text {a. }}$ Data are Mean \pm SD

b. Value in the parenthesis indicates the number of ASD afflicted children with reported problems over the total number of respondents. 
Am. J. Biochem. \& Biotech., 4 (2): 105-113, 2008

Table 3: SAA Metabolites Concentrations in Plasma

\begin{tabular}{cccc}
\hline Metabolite & Control $(\boldsymbol{\mu M})$ & ASD $(\boldsymbol{\mu M})$ & p \\
\hline Methionine & $40.7 \pm 19.1$ & 39.1 & $\mathrm{~ns}$ \\
S-Adenosylmethionine & $0.32 \pm 0.06$ & $0.38 \pm 0.09$ & $\mathrm{~ns}$ \\
S-Adenosylhomocysteine & $0.10 \pm 0.05$ & $0.13 \pm 0.08$ & $\mathrm{~ns}$ \\
Homocysteine & $6.7 \pm 2.9$ & $5.5 \pm 2.2$ & $\mathrm{~ns}$ \\
Cystathionine & $0.20 \pm 0.08$ & $0.22 \pm 0.07$ & $\mathrm{~ns}$ \\
Cysteine & $377.4 \pm 118$ & $375.9 \pm 61.0$ & $\mathrm{~ns}$ \\
GSH & $7.7 \pm 2.1$ & $7.3 \pm 2.3$ & $\mathrm{~ns}$ \\
Cysteinylglycine & $10.5 \pm 4.1$ & $17.5 \pm 6.8$ & 0.0008 \\
\hline
\end{tabular}

All values are Mean \pm SD

A

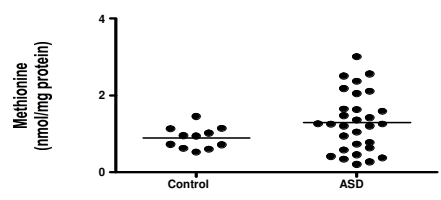

D

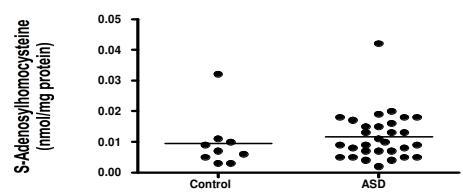

B

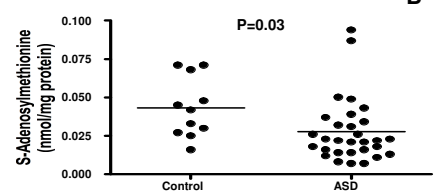

E

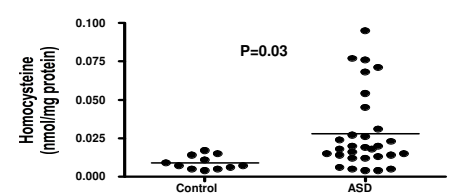

Fig 1: Transmethylation pathway metabolites in peripheral leukocytes of autistic and control children

Methionine (panel A), S-adenosylmethionine (panel B), SAM/SAH ratio (panel C), S-adenosylhomocysteine (panel D), and homocysteine (panel E) levels were determined in PBMC samples from healthy controls and children diagnosed with autism. Results show that PBMC from autistic individuals contained markedly lower levels of SAM and a decreased SAM/SAH ratio. Homocysteine levels were found to be significantly elevated in autistic individuals.

In contrast to plasma Cysgly, no other autismrelated changes in plasma SAA concentrations were detected (Table 2). This is in contrast to the finding by James and co-workers who show a marked ASD-related decrease in plasma SAA levels ${ }^{[16,17]}$. The discrepancies between our data and previously published data ${ }^{[16,17]}$ may be due to several factors. First, autism is highly heterogeneous and thus, our limited sample size may have not allowed us sufficient statistical power to detect subtle variations in plasma metabolite content. Second, potential differences in dietary intake, or nutritional supplementation by the participants in the different studies may have contributed to the discrepancies. Third, differences in the analytical techniques used could explain the discrepancies.

Autism-associated differences in SAA metabolism may be due to both genetic and environmental factors. SAA metabolism is heavily dependent on micronutrients such as folate, vitamin $\mathrm{B}_{6}$, and vitamin $\mathrm{B}_{12}$; thus insufficient dietary intake in ASD patients, but not in the controls, could contribute to our findings. Aside from environmental factors, genetics factors are likely contributing factor to our observations. It is worth noting that common single nucleotide polymorphisms of genes encoding enzymes involved in SAA metabolism have links to a number of developmental disorders including Down syndrome, cleft palate, and neural tube defects ${ }^{[37-39]}$.

Using a sensitive LC/MS/MS method, we detected significant changes in leukocyte SAA metabolism in autistic children. Interestingly, these changes were not related to alterations in plasma SAA availability. While these findings are intriguing, given the relatively small sample size of our study cohort, it may not be valid to generalize our preliminary findings to larger populations. Moreover, these results do not speak of a cause and effect relationship between autism and inflammation or whether the altered SAA metabolism is the cause of the inflammation seen in ASD. Despite these caveats, our results suggest that altered immune cell metabolism should be investigated to determine its role in autism.

Additionally, if the observed metabolic changes are due to genetic differences, it is conceivable, though highly speculative, that analysis of metabolic profiles might prove to be a useful means to identify genetically 


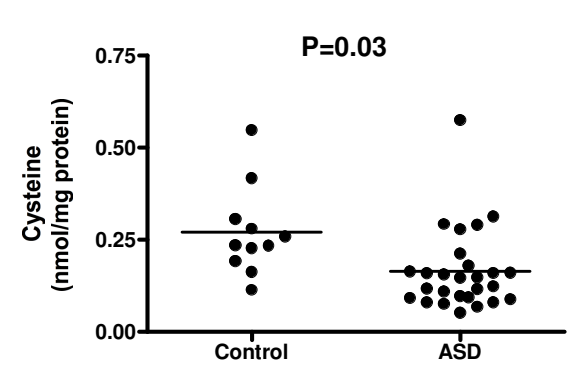

A

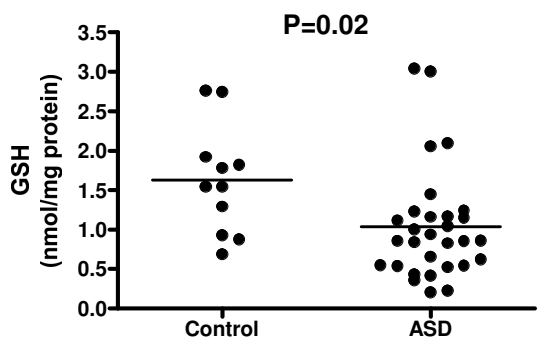

B

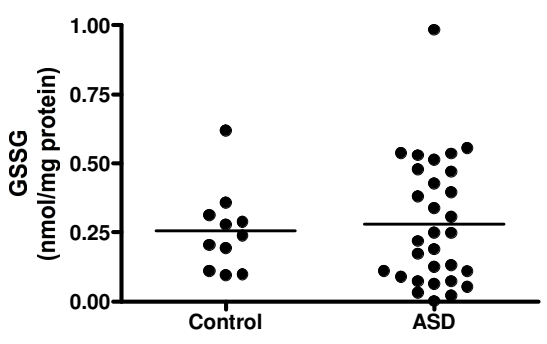

C
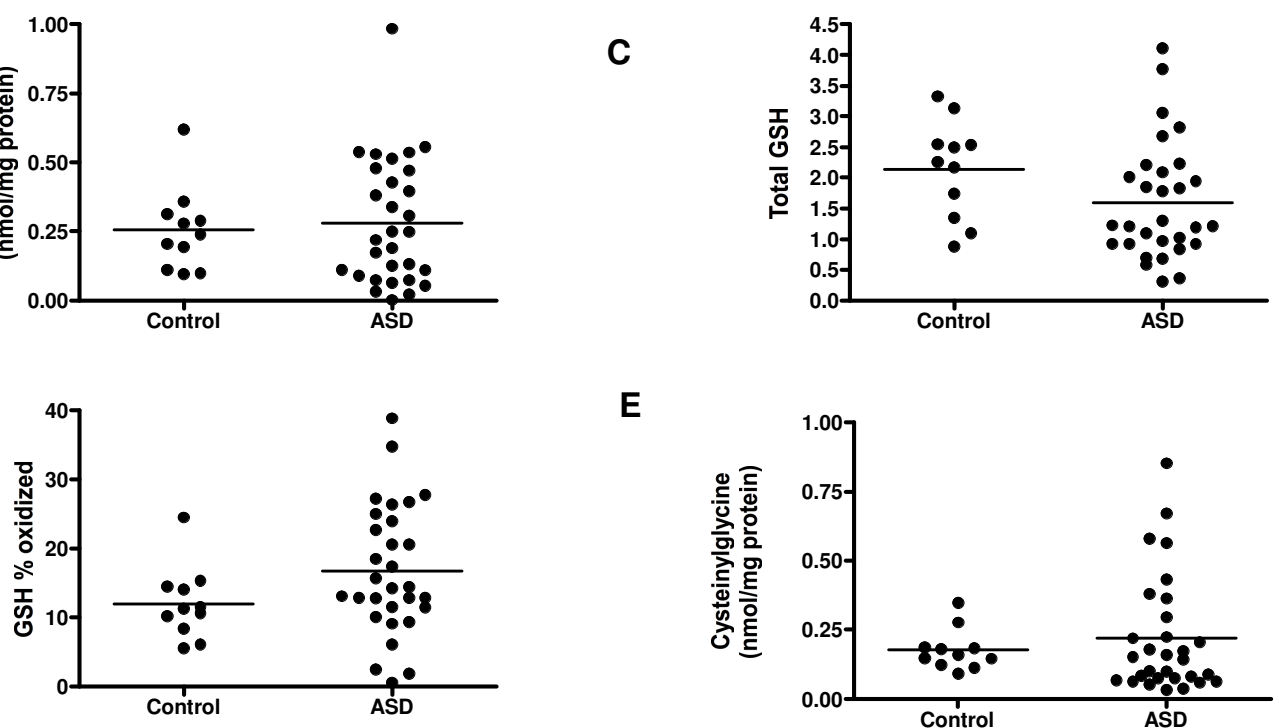

E

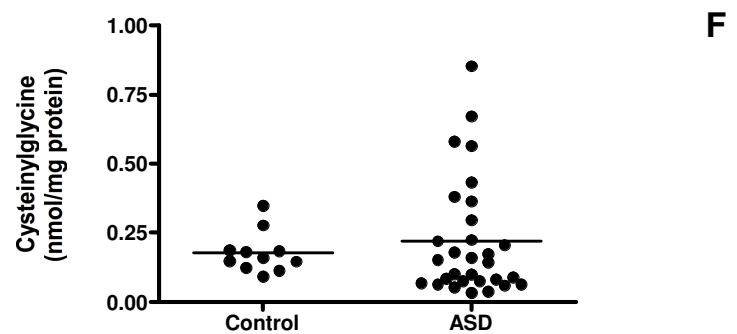

Fig 2: Peripheral leukocyte cysteine and glutathione content is lower in children with autism.

Leukocytes isolated from autistic children contained lower intracellular cysteine (panel A) and glutathione (GSH; panel B) concentrations than controls. However, no significant changes in GSSG (panel C), total GSH (GSH+2GSSG; panel D), or GSH redox state (panel E) were detected. Cysteinylglycine (panel F) concentrations in leukocytes was similar in both groups.

more homogeneous subgroups of autism. Expanded studies using much larger cohort of subjects would be needed, however, to validate such a metabolomic strategy for classifying subgroups of autism patients. If such a strategy proved successful, future studies might investigate possible correlations between different metabolic profiles and specific behaviors currently being used to diagnosis autism.

\section{ACKNOWLEDGEMENT}

The authors would like to thank Joyce McCann and John Nides for their critical reading of the manuscript and their comments. We would also like to acknowledge the financial support of Alexander and Bo MacInnis to the Pfeiffer Treatment Center.

\section{REFERENCES}

1. American Psychiatric Association, 1994. Diagnostic and statistical manual of mental disorders, $4^{\text {th }}$ edition. Washington, DC: American Psychiatric Press, Inc.

2. Autism and Developmental Disabilities Monitoring Network Surveillance Year 2002 Principal Investigators, and Centers for Disease Control and Prevention, 2007. Prevalence of autism spectrum disorders--autism and developmental disabilities monitoring network, 14 sites, United States, 2002. MMWR. Surveill. Summ., 56: 12-28.

3. Williams J.G., J.P. Higgins, and C.E. Brayne, 2006. Systematic review of prevalence studies of autism spectrum disorders. Arch. Dis. Child., 91: 8-15. 
4. Rutter M., 2005. Incidence of autism spectrum disorders: changes over time and their meaning. Acta. Paediatr., 94: 2-15.

5. Vargas D.L., C. Nascimbene, C. Krishnan, A.W. Zimmerman, and C.A. Pardo, 2005. Neuroglial activation and neuroinflammation in the brain of patients with autism. Ann. Neurol., 57: 67-81.

6. Warren R.P., N.C. Margaretten, N.C. Pace, and A. Foster, 1986. Immune abnormalities in patients with autism. J. Autism. Dev. Disord., 16: 189-197.

7. Jyonouchi H., S. Sun, and H. Le, 2001. Proinflammatory and regulatory cytokine production associated with innate and adaptive immune responses in children with autism spectrum disorders and developmental regression. J. Neuroimmunol., 120: 170-179.

8. Jyonouchi H., S. Sun, and N. Itokazu, 2002. Innate immunity associated with inflammatory responses and cytokine production against common dietary proteins in patients with autism spectrum disorder. Neuropsychobiology., 46: 76-84.

9. Jyonouchi H., L. Geng, A. Ruby, and B. Zimmerman-Bier, 2005. Dysregulated innate immune responses in young children with autism spectrum disorders: their relationship to gastrointestinal symptoms and dietary intervention. Neuropsychobiology., 51: 77-85.

10. Ashwood P., and A.J. Wakefield, 2006. Immune activation of peripheral blood and mucosal CD3+ lymphocyte cytokine profiles in children with autism and gastrointestinal symptoms. J. Neuroimmunol., 173: 126-134.

11. Molloy C.A., A.L. Morrow, J. Meinzen-Derr, K. Schleifer, K. Dienger, P. Manning-Courtney, M. Altaye, and M. Wills-Karp, 2006. Elevated cytokine levels in children with autism spectrum disorder. J. Neuroimmunol., 172: 198-205.

12. Griendling K.K., D. Sorescu, and M. Ushio-Fukai, 2000. NAD(P)H oxidase: role in cardiovascular biology and disease. Circ. Res., 86: 494-501.

13. Hughes G., M.P. Murphy, and E.C. Ledgerwood, 2005. Mitochondrial reactive oxygen species regulate the temporal activation of nuclear factor kappaB to modulate tumour necrosis factorinduced apoptosis: evidence from mitochondriatargeted antioxidants. Biochem. J., 389: 83-89.

14. Chauhan A., V. Chauhan, W.T. Brown, and I. Cohen, 2004. Oxidative stress in autism: increased lipid peroxidation and reduced serum levels of ceruloplasmin and transferrin--the antioxidant proteins. Life. Sci., 75: 2539-2549.
15. Ming X., T.P. Stein, M. Brimacombe, W.G. Johnson, G.H. Lambert, and G.C. Wagner, 2005. Increased excretion of a lipid peroxidation biomarker in autism. Prostaglandins. Leukot. Essent. Fatty. Acids., 73: 379-384.

16. James S.J., P. Cutler, S. Melnyk, S. Jernigan, L. Janak, D.W. Gaylor, and J.A. Neubrander, 2004. Metabolic biomarkers of increased oxidative stress and impaired methylation capacity in children with autism. Am. J. Clin. Nutr., 80: 1611-1617.

17. James S.J., S. Melnyk, S. Jernigan, M.A. Cleves, C.H. Halsted, D.H. Wong, P. Cutler, K. Bock, M. Boris, J.J. Bradstreet, S.M. Baker, and D.W. Gaylor, 2006. Metabolic endophenotype and related genotypes are associated with oxidative stress in children with autism. Am. J. Med. Genet. B. Neuropsychiatr. Genet., 141: 947-956.

18. Chauhan A., and V. Chauhan, 2006. Oxidative stress in autism. Pathophysiology., 13: 171-181.

19. McGinnis W.R., 2004. Oxidative stress in autism. Altern. Ther. Health. Med., 10: 22-36.

20. Grimble R.F., 2006. The effects of sulfur amino acid intake on immune function in humans. J. Nutr., 136: 1660S-1665S.

21. Kavanagh T.J., A. Grossmann, E.P. Jaecks, J.C. Jinneman, D.L. Eaton, G.M. Martin, and P.S. Rabinovitch, 1990. Proliferative capacity of human peripheral blood lymphocytes sorted on the basis of glutathione content. J. Cell. Physiol., 145: 472-480.

22. Roozendaal R., H.F. Kauffman, A.J. Dijkhuis, E.T. Ommen, D.S. Postma, J.G. de Monchy, and E. Vellenga, 2002. Interaction between nitric oxide and subsets of human $\mathrm{T}$ lymphocytes with differences in glutathione metabolism. Immunology., 107: 334-339.

23. Garg S., V. Vitvitsky, H.E. Gendelman, and R. Banerjee, 2006. Monocyte Differentiation, Activation, and Mycobacterial Killing Are Linked to Transsulfuration-dependent Redox Metabolism. J. Biol. Chem., 281: 38712-38720.

24. Pas sca S.P., B. Nemes, L. Vlase, C.E. Gagyi, E. Dronca, A.C. Miu, and M. Dronca, 2006. High levels of homocysteine and low serum paraoxonase 1 arylesterase activity in children with autism. Life. Sci., 78: 2244-2248. 
25. Yao Y., W.J. Walsh, W.R. McGinnis, and D. Praticò, 2006. Altered vascular phenotype in autism: correlation with oxidative stress. Arch. Neurol., 63: 1161-1164.

26. Husek P., P. Matucha, A. Vránková, and P. Simek, 2003. Simple plasma work-up for a fast chromatographic analysis of homocysteine, cysteine, methionine and aromatic amino acids. J. Chromatogr. B. Analyt. Technol. Biomed. Life. Sci., 789: 311-322.

27. LeGros H.L., A.M. Geller, and M. Kotb, 1997. Differential regulation of methionine adenosyltransferase in superantigen and mitogen stimulated human T lymphocytes. J. Biol. Chem., 272: 16040-16047.

28. German D.C., C.A. Bloch, and N.M. Kredich, 1983. Measurements of S-adenosylmethionine and Lhomocysteine metabolism in cultured human lymphoid cells. J. Biol. Chem., 258: 10997-11003.

29. Morris D.R., J.L. Degen, O.E. Oleinik, and C.E. Seyfried, 1981. Regulation of the level of Sadenosylmethionine decarboxylase during lymphocyte mitogenesis. Med. Biol., 59: 314-319.

30. Barbato J.C., O. Catanescu, K. Murray, P.M. DiBello, and D.W. Jacobsen, 2007. Targeting of metallothionein by L-homocysteine: a novel mechanism for disruption of zinc and redox homeostasis. Arterioscler. Thromb. Vasc. Biol., 27: 49-54.

31. Zhang Q., X. Zeng, J. Guo, and X. Wang, 2002. Oxidant stress mechanism of homocysteine potentiating Con A-induced proliferation in murine splenic T lymphocytes. Cardiovasc. Res., 53: 10351042.
32. Zhang Q., X. Zeng, J. Guo, and X. Wang, 2001. Effects of homocysteine on murine splenic B lymphocyte proliferation and its signal transduction mechanism. Cardiovasc. Res., 52: 328-336.

33. Ashwood P., and J. Van de Water, 2004. A review of autism and the immune response. Clin. Dev. Immunol., 11: 165-174.

34. Ashwood P., and J. Van de Water, 2004. Is autism an autoimmune disease? Autoimmun. Rev., 3: 557562.

35. Lee D.H., and D.R. Jacobs, 2005. Association between serum gamma-glutamyltransferase and Creactive protein. Atherosclerosis., 178: 327-330.

36. Bo S., R. Gambino, M. Durazzo, S. Guidi, E. Tiozzo, F. Ghione, L. Gentile, M. Cassader, and G.F. Pagano, 2005. Associations between gammaglutamyl transferase, metabolic abnormalities and inflammation in healthy subjects from a population-based cohort: a possible implication for oxidative stress. World. J. Gastroenterol., 11: 71097117.

38. Hobbs C.A., S.L. Sherman, P. Yi, S.E. Hopkins, C.P. Torfs, R.J. Hine, M. Pogribna, R. Rozen, and S.J. James, 2000. Polymorphisms in genes involved in folate metabolism as maternal risk factors for Down syndrome. Am. J. Hum. Genet., 67: 623-630.

39. Pezzetti F., M. Martinelli, L. Scapoli, F. Carinci, A. Palmieri, J. Marchesini, P. Carinci, E. Caramelli, R. Rullo, F. Gombos, and M. Tognon, 2004. Maternal MTHFR variant forms increase the risk in offspring of isolated nonsyndromic cleft lip with or without cleft palate. Hum. Mutat., 24: 104-105. 